257 (1995); M. S. Swanson and R. R. Isberg, Infect. Immun. 64, 2585 (1995).

14. P. Scholz et al., Gene 75, 271 (1989); J. Frey, M. M. Bagdasarian, M. Bagdasarian, ibid. 113, 101 (1992).

15. B. J. Bachman, in E. coli and Salmonella typhimurium, F. C. Neidhardt et al., Eds. (American Society for Microbiology, Washington, DC, 1987), pp. 1190-1219; J. E. Kelleher and E. A. Raleigh, J. Bacteriol. 173, 5220 (1991).

16. C. A. Lee, Infect. Agents Dis. 5, 1 (1996); J. Mecsas and E. J. Strauss, Emerg. Infect. Dis. 2, 271 (1996).

17. G. P. C. Salmond, Annu. Rev. Phytopathol. 32, 181
(1994); S. C. Winans, D. L. Burns, P. J. Christie, Trends Microbiol. 4, 64 (1996).

18. G. A. Kuldau, G. De Vos, J. Owen, G. McCaffrey, P. Zambryski, Mol. Gen. Genetics 221, 256 (1990); P. M. Rogowsky et al., Plasmid 23, 85 (1990); P. J. Christie, J. Bacteriol. 179, 3085 (1997).

19. V. Buchanan-Wollaston, J. E. Passiatore, F. Cannon, Nature 328, 172 (1987); A. Beijersbergen, A. D. Dulk-Ras, R. A. Schilperoort, P. J. Hooykaas, Science 256, 1324 (1992); K. J. Fullner, J. C. Lara, E. W. Nester, ibid. 273, 1107 (1996).

20. A. A. Weiss, F. D. Johnson, D. L. Burns, Proc. Natl. Acad. Sci. U.S.A. 90, 2970 (1993).
21. N. Ishiguro and G. Sato, J. Bacteriol. 164, 977 (1985); M. Sasatsu, T. K. Misra, L. Chu, R. Laddaga, S. Silver, ibid., p. 983.

22. G. Segal and H. A. Shuman, Infect. Immun. 65, 5057 (1997).

23. We thank M. Malamy and D. Cook for helpful suggestions and M. Bagdasarian for supplying the $\Delta$ oriT RSF1010 plasmid. Supported by a Medical Foundation grant to J.P.V. R.R.I. is an associate investigator of the Howard Hughes Medical Institute.

7 October 1997; accepted 16 December 1997

\title{
Meiotic Synapsis in the Absence of Recombination
}

\author{
Kim S. McKim, Becky L. Green-Marroquin, \\ Jeff J. Sekelsky, Gregory Chin, Carrie Steinberg, \\ Rita Khodosh, R. Scott Hawley
}

\begin{abstract}
Although in Saccharomyces cerevisiae the initiation of meiotic recombination, as indicated by double-strand break formation, appears to be functionally linked to the initiation of synapsis, meiotic chromosome synapsis in Drosophila females occurs in the absence of meiotic exchange. Electron microscopy of oocytes from females homozygous for either of two meiotic mutants (mei-W68 and mei-P22), which eliminate both meiotic crossing over and gene conversion, revealed normal synaptonemal complex formation. Thus, synapsis in Drosophila is independent of meiotic recombination, consistent with a model in which synapsis is required for the initiation of meiotic recombination. Furthermore, the basic processes of early meiosis may have different functional or temporal relations, or both, in yeast and Drosophila.
\end{abstract}

In the classical view of meiosis, homologous chromosome synapsis, as indicated by the formation of an elaborate ribbonlike structure called the synaptonemal complex (SC), was thought to be the first and primary event of meiotic prophase, essential for the initiation of meiotic recombination (1). Studies in Saccharomyces cerevisiae, however, have created a different view of the meiotic process in which the initiation of recombination, as evidenced by a doublestrand break (DSB), precedes the initiation of synapsis $(2,3)$. Three lines of evidence support this view of early meiotic prophase in yeast. First, the initiating event of meiotic recombination, the formation of a DSB, appears before SC formation (4). Second, meiotic mutants that either fail to create DSBs or to process DSBs to make single-stranded tails prevent the formation of a mature SC (2). Third, some mutants allow high levels of meiotic recombination but prevent the production of a mature SC (5). These data are consistent with a model in which single-stranded DNA generated by a DSB carries out a homology search required for synapsis and $\mathrm{SC}$ formation. In contrast, synapsis is not an absolute prereq-

Section of Molecular and Cellular Biology, University of California, Davis, CA 95616, USA. uisite for either the initiation (6) or completion of meiotic recombination (7).

To assess the relation between synapsis and the initiation of recombination in Drosophila oocytes, we examined both recombination and SC formation in oocytes homozygous for either of two null-recombination mutations. The mei-W68 and mei-P22 (8) mutants prevent the initiation of meiotic recombination as defined by four independent assays: (i) reduction or elimination of meiotic gene conversion; (ii) elimination of meiotic crossing over, as assayed by measuring either intragenic crossing over or the frequency of meiotic crossing over along entire chromosome arms; (iii) lack of doublestrand DNA breaks that persist into metaphase or anaphase I; and (iv) failure to produce either early or late recombination nodules (RNs).

To assay the effects of the mei-W68 and mei-P22 mutations on meiotic crossing over, we examined intragenic recombination at the rosy locus (9). No gene conversion events or intragenic crossovers were observed among the progeny of mei-W68 or mei-P22 females (Table 1 and Fig. 1). Compared to controls, the frequency of both intragenic exchange and simple gene conversion was reduced by a factor of at least 30 to 40 and, most likely, was eliminated. A small reduction in gene conversion frequency was also observed in the mei-W68/+ females, suggesting a dosage effect.

The effect of these mutations on crossing over was also assayed by more conventional means. In both mei-W68 and mei-P22 mutant females, the frequency of crossing over along the entire $X$ and second chromosomes was reduced to less than $0.5 \%$ of normal. Moreover, the few crossover events that were observed tended to be recovered in clusters of identical recombinants among the progeny of single females, suggesting that they resulted from mitotic and not meiotic recombination events (10). The failure to observe meiotic recombination events in progeny of mei-W68 and mei-P22 mutant

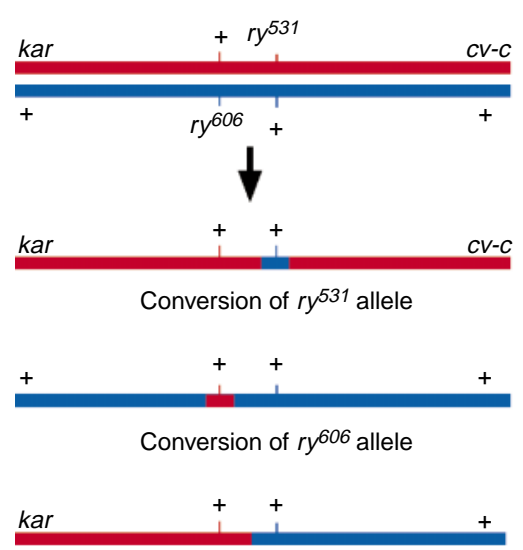

Crossover

Fig. 1. Gene conversion at the rosy locus (genetic map position 52.0). A schematic of the parental chromosomes and the three products of intragenic recombination that yield rosy ${ }^{+}$recombinants. The distances between the loci are not drawn to scale. The recombinants were classified as convertants or crossovers on the basis of the flanking mutations. In the mei-P22 experiment, the flanking markers were kar, an eye color mutant mapping $0.3 \mathrm{cM}$ to the left, and $c v-c$, a wing vein mutant mapping $2.1 \mathrm{cM}$ to the right of $r y$. In the mei-W68 experiment, Ace (52.5) or red (53.6) replaced $C V-C$ (54.1). The two rosy alleles used in this study, $r y^{531}$ and $r y^{606}$, were chosen because they are at opposite ends of the rosy gene, 3780 nucleotides and $0.012 \mathrm{cM}$ apart (29). The average length of conversion tracts in Drosophila is 885 base pairs (32), and therefore co-conversion events are expected to have a minimal effect on our experiments. 
Table 1. Intragenic recombination at the rosy locus in mei-P22, mei-W68, and control females.

\begin{tabular}{|c|c|c|c|c|c|}
\hline \multirow{2}{*}{ Genotype } & Crossover & $\begin{array}{l}\text { Conversion } \\
\text { of } r y^{606}\end{array}$ & $\begin{array}{l}\text { Conversion } \\
\text { of } r y^{531}\end{array}$ & $\begin{array}{l}\text { Zygotes } \\
\text { sampled }\end{array}$ & $\begin{array}{c}r y^{+} \\
\text {frequency }\end{array}$ \\
\hline & $\begin{array}{l}\mathrm{kar}^{+} \mathrm{ry}^{+} \mathrm{CV}-\mathrm{C} \text { or } \\
\mathrm{kar}^{+} r y^{+} \mathrm{Ace} \mathrm{e}^{126}\end{array}$ & $\begin{array}{l}k a r^{2} r y^{+} C v-c^{1} \text { or } \\
k a r^{2} r y^{+} A c e^{126}\end{array}$ & $\begin{array}{c}\mathrm{kar}^{+} \mathrm{ry}^{+} \mathrm{CV}-\mathrm{C}^{+} \\
\text {or kar+ } \mathrm{ry}^{+} \mathrm{Ace} \mathrm{C}^{+}\end{array}$ & $\left(\times 10^{5}\right)$ & $\left(\times 10^{-5}\right)$ \\
\hline thr ${ }^{1} \mathrm{kar}^{2} r y^{606} \mathrm{CV}-\mathrm{C}^{1} / r y^{531}$ & 20 & 9 & 5 & 5.7 & 5.96 \\
\hline mei-W68/+; kar ${ }^{2} r y^{606}$ Ace $e^{126} / r y^{531}$ & 9 & 1 & 3 & 3.88 & 3.35 \\
\hline mei-W68; kar² ry ${ }^{606}$ Ace ${ }^{126}$ or red/ry ${ }^{531 *}$ & 0 & 0 & 0 & 3.82 & $<0.9$ \\
\hline mei-P22 thr kar $^{2} r y^{606} \mathrm{cv}-\mathrm{c}^{1} / \mathrm{mei}^{-P 22} \mathrm{ry}^{531}$ & 0 & 0 & 0 & 5.6 & $<0.6$ \\
\hline
\end{tabular}

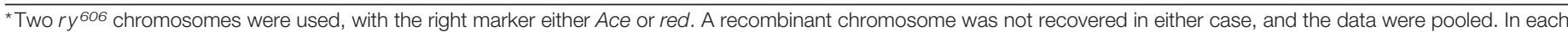
experiment, $1.91 \times 10^{5}$ progeny were screened.

oocytes is consistent with our failure to observe either early or late RNs in the oocytes of these females [(11) and see below].

Recombination events may be initiated in mei-P22 and mei-W68 oocytes, but then redirected into a pathway that results in sister-chromatid exchanges. We tested this possibility by examining the effects of these mutations on sister-chromatid exchange assayed by ring-chromosome loss. Sister-chromatid exchange within a ring chromosome results in the formation of a dicentric chromosome that is not transmissible. No excess of ring loss was observed in mei-P22 females compared with $+/+$ or $+/$ mei-P22 sisters (12).

The above data demonstrate that meiotic recombination events involving either homologous chromosomes or sister chromatids do not occur in mei-W68 and mei-P22 mutant oocytes. The ablation of gene conversion further demonstrates that mature recombination intermediates are also not formed. Based on three lines of evidence we

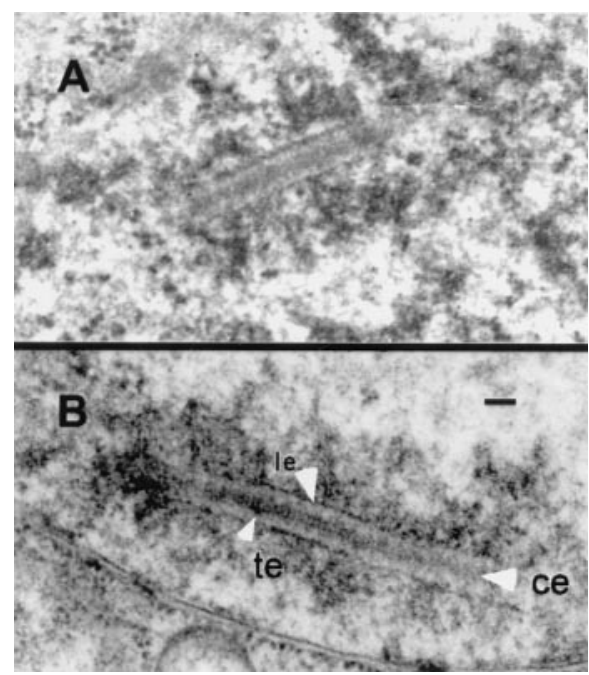

Fig. 2. Transmission electron microscopy of ovaries from females homozygous for mei-W68 and mei-P22. (A) Section from a mei-W68 female (16) and (B) a section from a mei-P22 female (33). The complete SC is visible in either mutant, including lateral elements (le), transverse elements (te), and central elements (ce). Bar, $100 \mathrm{~nm}$. can also rule out the possibility that DSBs are created in mei-W68 and mei-P22 mutant oocytes but left unrepaired. First, cytological analysis of meiotic chromosomes during metaphase and anaphase I from both meiW68 and mei-P22 mutant oocytes showed no evidence of chromosome fragmentation or of gapped chromosomes (13), as would be expected if the DSBs were not repaired (14). Second, there was no excess of nullo- $X$ oocytes relative to diplo-X oocytes among the progeny of either type of mutant female, and thus chromosome loss is not common (15). Finally, these females were no less fertile than expected, on the basis of expected frequencies of zygotic death due to aneuploidy, suggesting that there was no excessive dominant lethality from broken chromosomes in the oocytes. Although we cannot rule out the possibility that DSBs are made but are rapidly repaired in a manner that leaves no genetic trace, all available data

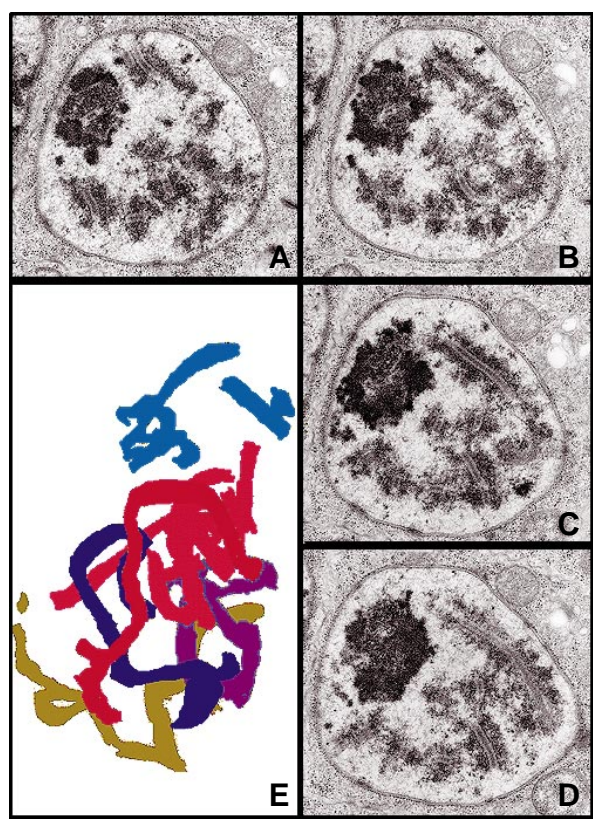

Fig. 3. (A to D) Consecutive sections from meiP22 females. (E) A tracing generated by superimposing all the sections from this nucleus, with each chromosome arm represented by a different color. argue that recombination is not initiated in these mutants.

Saccharomyces cerevisiae mutants exhibiting recombination defects as severe as those observed here do not make SC. To compare the effects of such a recombination defect in Drosophila oocytes, we examined SC formation in mei-W68 and mei-P22 oocytes. $\mathrm{SC}$ formation was analyzed by the reconstruction of serial sections examined by transmission electron microscopy. All of the pachytene nuclei from a single mei-W68 germarium plus several nuclei from two other germaria were reconstructed (Fig. 2A) (16). Three mei-P22 pachytene nuclei, two from a single germarium and one from another germarium, were completely reconstructed and 10 nuclei from several germaria were thoroughly examined (Figs. 2B and $3)$. Formation of the central and lateral elements and transverse filaments of the synaptonemal complex was normal in both mei-W68 and mei-P22 females. The width of the central region in euchromatic SC for both mei-W68 and mei-P22 was equal to that of wild-type $(109 \pm 8 \mathrm{~nm})(17)$. SC is continuous along each of the $X, 2 R, 2 L, 3 R$, $3 \mathrm{~L}$, and 4th chromosome arms (Fig. 3), and the age-adjusted euchromatic lengths of the SCs for the $X$ and $2 L$ are equal to those of wild type. There was no evidence for failed or nonhomologous synapsis. Consistent with the recombination phenotype, however, we failed to observe either early or late RNs in the mutants (11).

Meiotic progression in these mutants also appeared to be normal. We observed the same progression of SC shortening and thickening as in wild type. Three mei-W68 germaria were examined in detail (16), and in all respects the timing of developmental events was indistinguishable from that in wild type (17). In particular, there was no delay in reaching full pachytene. In meiW68 germaria there was an average of $1.5 \pm$ 1.7 16-cell cysts in pre-pachytene (16), compared with $1.3 \pm 0.9$ and $2.0 \pm 1.8$ in two different wild-type samples (18).

We conclude that, in Drosophila melanogaster oocytes, SC can form in the absence of detectable recombination events. Moreover, 
classical cytogenetic studies argue that in Drosophila oocytes it is synapsis that is required to initiate recombination, and not vice versa. For example, in D. melanogaster, Caenorhabditis elegans, maize and a variety of other organisms, heterozygosity for a translocation breakpoint substantially reduces crossing over for large regions surrounding that breakpoint (19). In yeast, however, similar rearrangements have little or no effect on the frequency of meiotic recombination in the vicinity of the breakpoint (20). Similarly, although small regions of ectopic DNA undergo recombination with their normally located homologous regions in yeast (7), the same is not true in Drosophila (21). Even large translocated regions only very rarely recombine or synapse (or both) with homologous intervals in Drosophila (22), worms (23), and mice (24).

We interpret these data to mean that large-scale synapsis is required to initiate meiotic recombination in Drosophila. Meiotic chromosome synapsis in yeast, which appears to require only a homology search done at a "gene-by-gene" level (25), may be representative of organisms with relatively small and noncomplex genomes. Indeed, such a strategy might well be disastrous in organisms with large amounts of dispersed repetitive DNA or with large dispersed gene families. We imagine that in higher organisms synapsis is required before the initiation of exchange both to prevent recombination events between homologous DNA sequences on nonhomologous chromosomes and to facilitate the nonrandom positioning of exchanges along the arms of meiotic chromosomes.

\section{REFERENCES AND NOTES}

1. R. S. Hawley and T. Arbel, Cell 72, 301 (1993).

2. G. S. Roeder, Proc. Natl. Acad. Sci. U.S.A 92, 10450 (1995).

3. N. Kleckner, ibid. 93, 8167 (1996).

4. R. Padmore, L. Cao, N. Kleckner, Cell 66, 1239 (1991).

5. B. Rockmill and G. S. Roeder, Genetics 126, 563 (1990); M. Sym and G. S. Roeder, Cell 79, 283 (1994).

6. B. de Massy, F. Baudat, A. Nicolas, Proc. Natl. Acad. Sci. U.S.A. 91, 11929 (1994); L. A. Gilbertson and F. W. Stahl, ibid., p. 11934

7. S. Jinks-Robertson and T. D. Petes, Genetics 114 731 (1986); M. Lichten, R. H. Borts, J. E. Haber, ibid. 115, 233 (1987).

8. The isolation and initial characterization of the second chromosome recessive mutation, mei-W68, was performed by B. S. Baker (26). The allele studied here is the more severe of the two existing alleles. The third chromosomal recessive mutant, mei-P22, was isolated in a large scale P-element mutagenesis screen for mutants with a high frequency of X-chromosome nondisjunction in the female germline (27). Subsequent analysis has shown that this mutant is caused by the insertion of a P-element (28).

9. Because of the low frequency of meiotic recombination in small intervals, gene conversion events in D. melanogaster are rare. The rosy system facilitates the isolation of intragenic recombinants, including gene conversions, because of a selection system for wild-type recombi- nants (29). Females with the genotypes shown in Table 1 were crossed to either TM2, ry/MKRS, ry kar males as virgins, or as nonvirgins to their phenotypically rosy brothers. These crosses were done in bottles with 30 females and 30 males for the controls, or 50 females and 50 males for the meiotic mutants. The flies were transferred to new bottles every 3 days at which time $0.75 \mathrm{ml}$ of $0.2 \%$ purine (Sigma) was added to the media. A total of five broods were set. To estimate the total number of progeny scored in each experiment, all the progeny were scored from the $\sim 5 \%$ of the bottles that were not given the purine treatment.

10. An exact estimate of the frequency of meiotic exchange in mei-W68 and mei-P22 mutants was complicated by the presence of premeiotic-exchange events. This problem is illustrated by an example with mei-P22. In our analysis of $\mathrm{X}$-chromosome crossing over in females homozygous for mei-P22, 25 crossovers were observed among 1935 regular progeny. Twenty-three of the crossovers occurred in clusters (one cluster of 18 identical recombinants and one cluster of 5 identica recombinants). Similar results were obtained on the left arm of chromosome 2 and in two sets of data regarding the effect of the mei-W68 mutation on second chromosome crossing over. Those recombinants arising in clusters can be ascribed to premeiotic (that is mitotic) recombination events, and thus the frequency of meiotic exchange in mei-P22 and mei-W68 females is either zero or very close to zero. In support of this conclusion, in males, which are normally achiasmatic, the mei-W68 mutation caused an increase in the spontaneous crossover frequency to an amount similar to that in mei-W68 females (30). Although the clusters of recombinants from mei-P22 females were larger than from mei-W68, the similarity of the mei-P22 and mei-W68 meiotic phenotypes (effects on gene conversion, and reductions in crossing over combined with increases in clustered events and their effects on SC formation) suggests that any differences between mei-P22 and mei-W68 in the production of crossover progeny are probably the result of differences in their effects on mitotic recombination.

11. Of the nine mei-W68 nuclei that were in the right developmental stage to have late RNs (between the onset of organelle passage through the ring canals and overt oocyte determination), no RNs were observed (16), despite the fact that with an average number of late nodules of 3.37 per nucleus (18) about 30 were expected. Similarly, no RNs were observed in three mei-P22 oocytes examined (16), although 10 were expected. No early RNs were observed in the four mei-W68 nuclei at the stage expected to display early RNs (that is, just before and just after the onset of organellar passage), when 11 would have been expected. The absence of RNs in mei-W68 and mei-P22 oocytes is concordant with the null-recombination phenotype of these mutants and is consistent with the hypothesis that neither mutant initiates recombination events.

12. In this experiment ring loss was measured by crossing $R(1) w^{v c / F M 7}$ females to $y w / y^{+} Y$ males, where $R(1) w^{v c}$ is a ring $X$ chromosome, $F M 7$ is a multiply inverted $X$ chromosome balancer chromosome that strongly suppresses $\mathrm{X}$-chromosomal exchange, and $y^{+} Y$ is a marked $Y$ chromosome. Ring recovery was assayed by comparing the frequency of $R(1) w^{v c} / y w$ female progeny with that of the corresponding FM7/y $w$ sisters. In the case of control females the Ring/Rod(FM 7 ) ratio was $0.854(n=2731)$, whereas in mei-P22 females the Ring/Rod ratio was increased to $1.079(n=659)$. Thus, rather than reducing recovery of the ring chromosome, as might be expected if mei-P22 oocytes exhibited an increased frequency of sister-chromatid exchange, the mei-P22 mutation appears to increase the frequency of ring recovery. A very similar set of results was obtained with a second null-recombination mutant $c(3) G^{17}$ in a similar experiment. Using a different ring-X chromosome [R(1)2], Hall observed a Ring/Rod ratio of 0.755 ( $n$ $=7552)$ in controls and an elevated ratio of $0.894(n=$ 5355) in c(3)G females (31). One reasonable explanation for these observations is that the reduced ring recovery observed in the two control experiments reflects the background frequency of meiotic sister-chromatid exchange, and that mei-P22 and $c(3) G$ actually inhibit these sister-chromatid exchange events, as they do interhomolog events, and in doing so increase the transmissibility of the ring-X chromosome.

13. K. S. McKim, J. K. Jang, R. S. Hawley, unpublished results.

14. C. W. Hinton and J. C. Lucchesi, Genetics 45, 87 (1960).

15. Among a total of 709 progeny from mei-P22 homozygous females, $11.6 \%$ were nullo-X exceptions and $18.3 \%$ diplo-X exceptions. Among a total of 1024 progeny from mei-W68 homozygous females, $20.7 \%$ were nullo-X exceptions and $18.0 \%$ diplo-X exceptions.

16. A. T. C. Carpenter, personal communication.

17. _ Chromosoma 51, 157 (1975).

18. —_ Genetics 92, 511 (1979); Chromosoma 75 , 259 (1979); Ciba Found. Symp. 182223 (1994).

19. P. A. Roberts, Genetics 65, 429 (1970); ibid. 71, 401 (1972); R. S. Hawley, ibid. 94, 625 (1980); R. E. Rosenbluth and D. L. Baillie, ibid. 99, 415 (1981); C. R. Burnham, J. T. Stout, W. H. Weinheimer, R. V. Kowles, R. L. Phillips, ibid. 71, 111 (1972); M. P. Maguire, J. Theor. Biol. 106, 605 (1984).

20. F. Sherman, C. Helms, Genetics 88, 689 (1978); J. E. Haber, W.-Y. Leung, R. H. Borts, M. Lichten, Proc. Natl. Acad. Sci. U.S.A. 88, 1120 (1991); A. S. H. Goldman and M. Lichten, Genetics 144, 43 (1996).

21. R. Hipeau-Jacquotte, D. L. Brutlag, F. Brégégère, Mol. Gen. Genet. 220, 140 (1989).

22. L. Craymer, Genetics 99, 75 (1981); ibid. 108, 573 (1984).

23. A. M. Rose, D. L. Baillie, J. Curran, Mol. Gen. Genet. 195, 52 (1984); K. S. McKim, A. M. Howell, A. M. Rose, Genetics 120, 987 (1988); K. S. McKim, K. Peters, A. M. Rose, ibid. 134, 749 (1993).

24. P. B. Moens, J. A. M. Heddle, H. H. Q. Heng, Genome 40, 770 (1997).

25. N. Kleckner, R. Padmore, D. K. Bishop, Cold Spring Harbor Symp. Quant. Biol. 56, 729 (1991).

26. B. S. Baker, personal communication.

27. R. S. Hawley et al., unpublished results.

28. K. S. McKim, unpublished results.

29. A. J. Hilliker, S. Clark, A. Chovnick, Genetics 129 , 779 (1991)

30. T. Lutken and B. S. Baker, Mutat. Res. 61, 221 (1979)

31. J. Hall, Drosophila Information Service 52, 142 (1977).

32. D. Curtis and W. Bender, Genetics 127, 739 (1991).

33. Ovaries from females homozygous for mei-P22 were removed in a $0.7 \% \mathrm{NaCl}$ solution as described by Carpenter (18) with minor modifications; 4\% glutaraldehyde in cacodylate buffer $(\mathrm{pH}$ 7.0) was used instead of Karnovsky's fixative, and instead of storing overnight in buffer, the ovaries were rinsed and immediately placed in $2 \%$ osmium tetroxide in cacodylate buffer ( $\mathrm{pH} 7.0)$ for 1.5 hours. Ovaries were thoroughly rinsed with distilled $\mathrm{H}_{2} \mathrm{O}$, dehydrated with ethanol to $70 \%$, and en bloc stained with $2 \%$ uranyl acetate dissolved in $70 \%$ ethanol overnight. Ovaries were embedded in Spurr's resin instead of EponAraldite. Whole ovaries were flat-embedded with the ovarioles positioned so that the long axis of the germarium was parallel to the cutting plane. The germarium end of the block was serial sectioned with a thickness of $100 \mathrm{~nm}$ per section. The sections were collected on formvar, carbon-coated copper singleslot grids ( $1 \mathrm{~mm}$ by $2 \mathrm{~mm}$ ). The sections were then poststained with uranyl acetate (in 50\% ethanol) for $20 \mathrm{~min}$ and concentrated lead citrate for $30 \mathrm{~s}$, with thorough rinsing after each staining. The sections were examined by electron microscopy (Philips EM410) and photographed at low magnification. When the cells with four ring canals were located, they were photographed at a magnification of 14,000.

34. We are grateful to A. T. C. Carpenter for advice throughout all aspects of this study and for supplying the EM data for SC formation in mei-W68 mutants. We also thank B. S. Baker for data on the initial genetic characterization of mei-W68; J. Haber, N. Kleckner, and T. Petes for critical reading of the manuscript; and T. Arbel, W. Hurley, and R. French for assistance in the isolation of mei-P22. K.S.M. was supported by a fellowship from the Medical Research Council (Canada). Supported by a grant from the $\mathrm{NIH}$ to R.S.H.

14 August 1997; accepted 18 December 1997 